\title{
AOR
}

Selected Papers of \#AoIR2019:

The $20^{\text {th }}$ Annual Conference of the

Association of Internet Researchers

Brisbane, Australia / 2-5 October 2019

\section{Generational differences in social media use, gender identity, and sexuality among young LGBTIQ+ people in Australia}

\author{
Brady Robards \\ Monash University \\ Brendan Churchill \\ University of Melbourne \\ Son Vivienne \\ RMIT University \\ Benjamin Hanckel \\ Kings College London \\ Paul Byron \\ Swinburne University
}

Over the past twenty to thirty years, the Internet has come to serve as a key channel for communicating, connecting, and engaging in civic participation. For lesbian, gay, bisexual, transgender, intersex, and queer or questioning (LGBTIQ+) people, who continue to experience disproportionate health risks and high rates of discrimination (Leonard et al. 2012), the significance of the internet as a social resource is further magnified (Hanckel and Morris 2014).

While digital social spaces have evolved, many of the motivations for using these platforms remain the same. Different platforms offer different opportunities to connect with queer peers and others, for discussing, documenting and exploring sexuality away from heteronormative spaces (Hillier et al. 2010, O'Neill 2014). From text-based 'virtual' worlds and discussion boards of years gone by, through to contemporary sites and apps such as Facebook, Instagram, Twitter, Snapchat, Tumblr, YouTube, Reddit, Tinder, Grindr and Her, the digital social media landscape is now more complex than ever. What new challenges and opportunities does this evolution present?

Suggested Citation (APA): Robards, B., Churchill, B., Vivienne, S. \& Byron, P. (2019, October 2-5). Social media use among young LGBTIQ+ people in Australia. Paper presented at AolR 2019: The 20th Annual Conference of the Association of Internet Researchers. Brisbane, Australia: AolR. Retrieved from http://spir.aoir.org. 
This paper draws on data from the Scrolling Beyond Binaries study, centred on a national survey of 1,304 young LGBTIQ+ Australians. We present key findings from the study in two parts: (a) differences across our four age cohorts of our young respondents $(16-20,21-25,26-30,30-35)$ in terms of their identities (gender and sexuality) and the social media platforms they use; and (b) the argument that the internet continues to be significant for our respondents for social connection and learning. In doing so, we explore the complex and evolving ways in which young LGBTIQ+ people use and thus (re)produce digital social spaces, returning to Nina Wakeford's (2000 [1997]) consideration of 'cyberqueer spaces'.

\section{Diverse Genders \& Sexualities}

Our two younger cohorts (16-20 and 21-25) were more likely than our older two cohorts to identify as non-binary when it comes to gender: $21 \%$ of our $16-20$ year old cohort, and $26 \%$ of our $21-25$ year old group, compared to $14 \%$ of the $26-30$ group $9 \%$ of the 31-35 group. In addition to providing gender options female (45\% of respondents overall), male (26\%), and non-binary (19\%), we also invited respondents to complete an open-ended field to name their own gender identity. $9 \%$ of our respondents made use of this field, with the most common responses being gender-fluid, agender and trans. Tracing the antecedents of non-binary identities across generations in archives or public records is thwarted by the fact that these categories have not previously been available, despite the likelihood that gender-diverse people have long existed. Through categorisation they are inevitably folded in normative forms. What is clear, however, is that 'cyberqueer' spaces (with Tumblr being a prime example) have become central for the forming of connections and the discovery of a common language, a sub-cultural knowledge, by which to describe the non-binary, fluid, and trans experiences of gender.

Like gender, when it comes to sexuality, our older respondents were more likely to identify with a more 'long-standing' and binary category like lesbian, gay, or homosexual: $48 \%$ for the $31-35$-year-old cohort, compared to $27 \%$ for the 16 -to-20year-old cohort. In fact, more of our youngest respondents (16-20) identified as bisexual than they did as lesbian or gay. As with gender diversity, a movement away from binary sexual identities is reflected in other recent Australian studies. Hillier et al. (2010, p. 27) found their female respondents identified as bisexual (42\%) rather than lesbian (39\%), and in Byron et al.'s study (2017, p. 17), more young people identified as bisexual $(32.3 \%)$ than lesbian $(21.5 \%)$ or gay (18.5\%). A large number of participants in Byron et al.'s (2017) study also identified as pansexual (19.1\%) and asexual (9.2\%).

\section{Differences in platform use}

There are also clear differences in patterns of social media use in our four age cohorts. Generally, the use of almost all platforms declines for each subsequent older cohort, with some exceptions. Facebook (97\%), YouTube (84\%) and Instagram (70\%) are commonly used platforms for all of our respondents. There were, however, significant differences in other platforms. There was an age gradient in the use of apps like Snapchat and Tumblr, where usage was highest among younger cohorts. Snapchat, for instance, was used by $80 \%$ of our 16 -to 20 -year-olds, but by just $34 \%$ of our 31 -to-35year-old cohort. Reddit use was also higher for the middle cohort (almost one-quarter of 
21-to-25-year-olds and $21 \%$ of 26-to 30-year-olds) but lower among our youngest and oldest cohorts (13\% of 16 -to-20-year-olds and $16 \%$ of 31 -to-35-year-olds).

There are also interesting patterns of use of hook-up and dating apps across our age cohorts. Overall, Tinder was the most popular, with $21 \%$ of respondents using it, followed by Grindr (11\%), OKCupid (7.5\%), Her (6\%), and Scruff (5\%). When we combine these apps and instead divide them into categories that allow for multiple sexual orientations or interests (like Tinder or OKCupid) versus apps that are more specific - for gay men or women (like Grindr, Her, Scruff, and Hornet) - there is an evident age gradient. The youngest cohorts in this study were more likely to use platforms such as Tinder, Happn and Bumble that allow for users to engage with more than one sex (e.g. Happn allows for users to 'find' men and women). In contrast, the older cohorts were more likely to use targeted hook-up/dating apps like Grindr or Her.

This significant difference in usage may reflect the historical development of hook-up and dating apps, where apps like Grindr and Scruff were amongst the first hookup/dating apps available, preceded by dating sites specifically for gay men, like Gaydar (see Light et al. 2008). This difference may also reflect the fluidity in sexuality and sexual preferences of users at younger ages, in which apps that allow for more 'choice' appear preferable. For us, one of the key points this age gradient points to is evidence of the changing patterns in the way young people describe their own gender and sexual identities, and how digital media might map to these changing conceptualisations and the evolution of cyberqueer spaces.

\section{References}

Byron, P., et al., 2017. 'You Learn from Each Other': LGBTIQ Young People's Mental Health Help-Seeking and the RAD Australia Online Directory. Sydney, NSW: Western Sydney University \& Young and

Hanckel, B. and Morris, A., 2014. Finding Community and Contesting Heteronormativity: Queer Young People's Engagement in an Australian Online Community. Journal of Youth Studies, 17 (7), 872-886. doi:10.1080/13676261.2013.878792

Hillier, L., et al., 2010. Writing Themselves in 3: The Third National Study on the Sexual Health and Wellbeing of Same Sex Attracted and Gender Questioning Young People. Melbourne, VIC: Australian Research Centre in Sex, Health and Society, La Trobe University.

Leonard, W., et al., 2012. Report. In: Private Lives 2: The second national survey of the health and wellbeing of GLBT Australians, Australian Research Centre in Sex, Health \& Society, LaTrobe University, 1-75, available online:

https://www.glhv.org.au/sites/default/files/PrivateLives2Report.pdf.

Light, B., Fletcher, G., and Adam, A., 2008. Gay Men, Gaydar and the Commodification of Difference. Information Technology \& People, 21 (3), 300-314. 
O'Neill, M., 2014. Transgender Youth and YouTube Videos: Self-Representation and Five Identifiable Trans Youth Narratives. In: C. Pullen, ed. Queer Youth and Media Cultures. New York, NY: Palgrave Macmillan, 34-45.

Wakeford, N., 2000 [1997]. Cyberqueer. In: D. Bell and B. M. Kennedy, eds. The Cybercultures Reader. Abington: Routledge, 401-415. 